\title{
When is radiation therapy in head and neck squamous cell carcinoma not indicated?
}

\author{
William M. Mendenhall • Primož Strojan • Carl E. Silver • \\ Randall P. Owen • Alessandra Rinaldo • Ashok R. Shaha • \\ Avraham Eisbruch · Robert P. Takes • Alfio Ferlito
}

Received: 30 September 2013/Accepted: 7 October 2013/Published online: 20 October 2013

(C) Springer-Verlag Berlin Heidelberg 2013

Head and neck squamous cell carcinoma (HNSCC) can be treated with surgery, radiotherapy (RT), and/or chemotherapy depending on the location and extent of the neoplasm. Treatment selection also depends on the goal of therapy (curative or palliative) and the expected efficacy weighed against the expected morbidity of the treatment. Moreover, it may be determined by the treatment modalities available, although all modalities will usually be available in most centers in developed countries. Earlystage HNSCCs can usually be treated with a single modality, whereas advanced-stage disease requires multimodality treatment. The choice of treatment modality will depend on factors including tumor site, existing and expected functionality, cosmetic outcome, age, performance status, the likelihood that adjuvant postoperative RT will be required, and the wishes of the patient. Following is a discussion of the curative-intent management of patients

The paper was written by members of the International Head and Neck Scientific Group (www.IHNSG.com).

W. M. Mendenhall

Department of Radiation Oncology, College of Medicine,

University of Florida, Gainesville, FL, USA

P. Strojan

Department of Radiation Oncology, Institute of Oncology,

Ljubljana, Slovenia

C. E. Silver

Departments of Surgery and Otolaryngology, Head and Neck Surgery, Albert Einstein College of Medicine, Montefiore Medical Center, New York, Bronx, USA

R. P. Owen

Division of Metabolic, Endocrine and Minimally Invasive Surgery, Department of Surgery, The Icahn School of Medicine at Mt. Sinai, New York, USA with mucosal HNSCC and situations where RT can be safely eliminated.

Surgery alone is the preferred treatment for patients with HNSCC when the probability of a complete resection is high (>85-90\%) and the functional and cosmetic outcome is acceptable. An important caveat is that a functional/ cosmetic outcome that is acceptable to the physician may or may not be acceptable to the patient. Other factors such as patient age and medical co-morbidities also influence the decision. Patients with human papilloma virus (HPV)positive oropharyngeal squamous cell carcinomas (SCCs) have a better prognosis than those with HPV-negative SCCs. It could be reasoned that these patients should be treated differently. Depending on extent of disease, surgery alone, transoral robotic surgery (TORS) in particular, could be an attractive option as this group of patients is usually of younger age. However, the question if and how patients should be treated differently depending on HPV status is the subject of trials now and no firm recommendation can be made at this point $[1,2]$. Although the adverse impacts

A. Rinaldo · A. Ferlito $(\square)$

ENT Clinic, University of Udine Piazzale S. Maria della Misericordia, 33100 Udine, Italy

e-mail: a.ferlito@uniud.it

A. R. Shaha

Department of Surgery, Memorial Sloan-Kettering Cancer Center, New York, USA

A. Eisbruch

Department of Radiation Oncology, University of Michigan,

Ann Arbor, Michigan, USA

R. P. Takes

Department of Otolaryngology, Head and Neck Surgery,

Radboud University Nijmegen Medical Center, Nijmegen,

The Netherlands 
of surgery are appreciated shortly after the operation, late RT toxicity may occur months to years after treatment. For example, swallowing difficulty resulting in aspiration and requiring a permanent gastrostomy may not become apparent for years after RT. There is also a risk, albeit quite low, of a radiation-induced cancer 7-10 years after RT. Therefore, surgery is preferred over RT, all things being equal, for younger patients with a long life expectancy. Some medical co-morbidities such as connective tissue diseases may also significantly increase the risk of late radiation toxicity. Patients with scleroderma, for example, are best treated with surgery rather than RT. Patients treated previously with RT for HNSCC have a high risk of a complication after an additional course of irradiation and are better treated surgically.

Following surgery, adjuvant postoperative RT may be withheld if the likelihood of a complete resection is high, particularly if an acceptable salvage option is available in the event of a local-regional recurrence. Pathologic factors that indicate the need for postoperative RT include the following: close ( $\leq 5 \mathrm{~mm}$ ) or positive margins, two or more positive nodes, extracapsular extension (ECE), invasion of the soft tissues of the neck, bone invasion, perineural invasion (PNI), and endothelial lined space invasion [3, 4]. Some indications are more ominous than others, such as positive margins and ECE which usually required the addition of concomitant adjuvant chemotherapy (chemoradiation). Other indications, such as close margins or early bone invasion, may not necessarily indicate postoperative RT. A 2-mm margin after a partial glossectomy is usually an indication for postoperative RT, whereas this would not be the case after a $\mathrm{CO}_{2}$ laser excision of an early vocal cord cancer.

Patients with the following clinically staged HNSCCs are generally considered for treatment with surgery alone: T1-T2N0 oral cavity, T1-T2N0 oropharynx [5, 6], T1N0 glottis limited to one vocal cord [7], and T1-T2N0 supraglottis [8]. Postoperative RT is added for the indications previously discussed but the intention in the treatment of these patients should be single modality treatment to avoid the morbidity of more than one treatment modality. Patients with a single pathologically positive node without ECE may be followed closely after surgery [4]. The above generalizations are subject to a number of caveats and many would generate a spirited discussion.

\section{Conclusion}

In conclusion, patients with T1-T2N0 SCCs are preferably treated with surgery alone depending on the primary site, the functional status and expected functional and cosmetic outcomes, and the medical condition of the patient. The decision on which treatment to give or not to give should ideally be made in multidisciplinary teams and based on the highest available levels of evidence from the literature. As information from the literature is often incomplete or conflicting, ultimately the question the attending physician(s) must ask in those cases is, "What would I do if it were me or a member of my family?"

\section{References}

1. Salazar CR, Smith RV, Garg MK, et al (2013) Human papillomavirus-associated head and neck squamous cell carcinoma survival: a comparison by tumor site and initial treatment. Head Neck Pathol. doi:10.1007/s12105-013-0486-4

2. Kofler B, Laban S, Busch CJ, Lorincz B, Knecht R (2013) New treatment strategies for HPV-positive head and neck cancer. Eur Arch Otorhinolaryngol. doi:10.1007/s00405-013-2603-0

3. Mendenhall WM, Amdur RJ, Hinerman RW, Villaret DB, Siemann DW (2003) Postoperative radiation therapy for squamous cell carcinoma of the head and neck. Am J Otolaryngol 24:41-50

4. Strojan P, Ferlito A, Langendijk JA, Silver CE (2012) Indications for radiotherapy after neck dissection. Head Neck 34:113-119

5. Haughey BH, Hinni ML, Salassa JR et al (2011) Transoral laser microsurgery as primary treatment for advanced-stage oropharyngeal cancer: a United States multicenter study. Head Neck 33:1683-1694

6. Weinstein GS, Quon H, O'Malley BW Jr, Kim GG, Cohen MA (2010) Selective neck dissection and deintensified postoperative radiation and chemotherapy for oropharyngeal cancer: a subset analysis of the University of Pennsylvania transoral robotic surgery trial. Laryngoscope 120:1749-1755

7. Mendenhall WM, Werning JW, Hinerman RW, Amdur RJ, Villaret DB (2004) Management of T1-T2 glottic carcinomas. Cancer 100:1786-1792

8. Hinerman RW, Mendenhall WM, Amdur RJ, Stringer SP, Villaret DB, Robbins KT (2002) Carcinoma of the supraglottic larynx: treatment results with radiotherapy alone or with planned neck dissection. Head Neck 24:456-467 